\title{
Cost Utility of Fractional Exhaled Nitric Oxide Monitoring for the Management of Children Asthma
}

\author{
jefferson buendia ${ }^{1}$, Ranniery Acuña-Cordero ${ }^{2}$, and Carlos Rodriguez-Martinez ${ }^{3}$ \\ ${ }^{1}$ University of Antioquia \\ ${ }^{2}$ Hospital Militar Central \\ ${ }^{3}$ School of Medicine, Universidad El Bosque
}

June 3, 2020

\begin{abstract}
Introduction Fractional exhaled nitric oxide (FeNO) is a simple, noninvasive measurement of airway inflammation with minimal discomfort to the patient and with results available with a few minutes. This study aimed to evaluate the cost-effectiveness of the Fractional exhaled nitric oxide in asthma. Methods A markov model was used to estimate the cost-utility of FeNO versus standard treatment (control group) an infant with mild to moderate allergic asthma. Cost data were obtained from a retrospective study on asthma from tertiary center, in Medellin, Colombia, while utilities were collected from the literature. The analysis was carried out from a societal perspective. Results The model showed that FeNO, was associated with lower total cost than SC (US $\$ 1333$ vs US $\$ 1452$ average cost per patient), and higher QALYs (0.93 vs. 0.92 average per patient); showing dominance. The probability that MS provides a more cost-effective use of resources compared with standard therapy exceeds 99\% for all willingness to pay thresholds Conclusion FeNO was cost-effective for the hospital treatment of an infant with infant with mild to moderate allergic asthma. Our study provides evidence that should be used by decision-makers to improve clinical practice guidelines and should be replicated to validate their results in other middle-income countries.
\end{abstract}

Cost Utility of Fractional Exhaled Nitric Oxide Monitoring for the Management of Children Asthma

Short running title: Fractional Exhaled Nitric Oxide in Asthma

Jefferson Antonio Buendía, MD,MSc, $\mathrm{PhD}^{1}$, Ranniery Acuña-Cordero $\mathrm{MD}^{2}$, Carlos E. Rodriguez-Martinez $\mathrm{MD}, \mathrm{MSc}, \mathrm{PhD}(\mathrm{c})^{3,4}$

Department of Pharmacology and Toxicology, School of Medicine, Research group in pharmacology and toxicology (INFARTO). Universidad de Antioquia, Medellín, Colombia.

Departamento de Neumología Pediátrica, Hospital Militar Central, Departamento de Pediatría, Facultad de Medicina, Universidad Militar Nueva Granada, Bogotá, Colombia

Department of Pediatrics, School of Medicine, Universidad Nacional de Colombia, Bogota, Colombia

Department of Pediatric Pulmonology and Pediatric Critical Care Medicine, School of Medicine, Universidad El Bosque, Bogota, Colombia

Corresponding author: Jefferson A. Buendía, MD, Ph.D., Facultad de Medicina, Universidad de Antioquia, Carrera 51D \#62-29, Tel: +574 2196046; Medellín, Colombia. E-mail: jefferson.buendia@gmail.com. E-mail: JAB: jefferson.buendia@gmail.com

\section{Introduction}


Fractional exhaled nitric oxide (FeNO) is a simple, noninvasive measurement of airway inflammation with minimal discomfort to the patient and with results available with a few minutes. This study aimed to evaluate the cost-effectiveness of the Fractional exhaled nitric oxide in asthma.

\section{Methods}

A markov model was used to estimate the cost-utility of FeNO versus standard treatment (control group) an infant with mild to moderate allergic asthma. Cost data were obtained from a retrospective study on asthma from tertiary center, in Medellin, Colombia, while utilities were collected from the literature. The analysis was carried out from a societal perspective.

\section{Results}

The model showed that FeNO, was associated with lower total cost than SC (US $\$ 1333$ vs US $\$ 1452$ average cost per patient), and higher QALYs (0.93 vs. 0.92 average per patient); showing dominance. The probability that MS provides a more cost-effective use of resources compared with standard therapy exceeds $99 \%$ for all willingness to pay thresholds

\section{Conclusion}

FeNO was cost-effective for the hospital treatment of an infant with infant with mild to moderate allergic asthma. Our study provides evidence that should be used by decision-makers to improve clinical practice guidelines and should be replicated to validate their results in other middle-income countries.

Keywords: health economics, public health, healthcare

\section{Introduction}

The high proportion of patients without adequate control of asthma generates permanent challenges worldwide. For example, in Latin America, only $2.4 \%$ of the patients with this disease have total asthma control (1). The periodic assessment and the early management of airway inflammation are the principal strategies, to prevent hospitalizations, recommended for international and local clinical guidelines $(2,3)$. In this sense, the frequent measure of airway inflammation during the follow up plays an important role to anticipate the exacerbations and optimize the use of biological and corticosteroids drugs.

Since early of the nineties, the measure of fractional exhaled nitric oxide (FeNO) has been used as a surrogate marker for type 2 airway inflammation(4). FeNO is a simple, noninvasive measurement of airway inflammation with minimal discomfort to the patient and with results available with a few minutes. FeNO correlates with airway eosinophilia in biopsy and bronchoalveolar lavage fluid (5). Indeed, a meta-analysis of eight clinical trials in children found that FeNO guided treatment reduced asthma exacerbations (6). The National Institute for Health and Care Excellence (NICE) since 2014 recommended FeNO testing in children when asthma diagnosis unclear. However, the routine used of FeNO in asthma, and in children, is not uniformly adopted by all countries, especially those developing countries.

The main barriers to adopting new technologies, from policymakers, are always doubts about their efficiency in scenarios with scarce health resources. Different economic evaluations of the use of FeNO during asthma management in developed countries have been demonstrated that FeNO monitoring to guide asthma management was cost-effective in Spain, Germany, UK, and US (7-10). In this paper, we aimed to evaluate the cost-utility of FeNO monitoring to guide asthma management in Children in a middle-income country from a societal perspective.

\section{Material and methods}

\section{Economic model}

A Markov simulation model with three mutually exclusive nonabsorbent states was used to compare the estimated cost and outcomes associated with asthma management using standard care (SC) or SC with 
FeNO monitoring to guide asthma management in Children (FeNO-SC) (Figure 1 ). This model and according to the natural history, have three health states were defined: "no symptoms", "suboptimal, control, no exacerbation", and "asthma exacerbation" with a cycle length of 1 week. All patients entering the model are children with no symptoms, diagnosed with mild to moderate allergic asthma, and receiving inhaled corticosteroids as maintenance therapy. The effectiveness outcome was the quality-adjusted life years (QALYs). The analysis was carried out from a societal perspective (included direct and indirect costs). The analytic horizon was 12 months. Given the short time horizon, no type of discount to costs or results was applied. The study protocol was reviewed and approved by the Institutional Review Board of University of Antioquia (No 18/2015).

\section{Intervention}

These were obtained from a systematic review of published randomized clinical trials and systematic reviews of randomized clinical trials (RCT). Searches of computerized databases (MEDLINE, EMBASE, CENTRAL, and LILACS) and references cited in published literature identified the potentially applicable studies. The structured literature searches in these databases were made using the following criteria: (asthma OR wheeze) and ("Nitric Oxide" [tiab] or FeNO or eNO "exhaled NO"[tiab]). The searches yielded 493 citations, and a total of 34 studies were examined in full text. Included studies comparing adjustment of asthma medications based on FeNO levels versus management based on clinical symptoms or current asthma guidelines or both. We excluded studies with the following comorbidities/ characteristics: eosinophilic bronchitis, asthma-related to underlying lung disease such as bronchiectasis and chronic obstructive pulmonary disease or diagnostic categories such as 'cough variant asthma' and 'wheezy bronchitis'. After applying these criteria, data from 8 studies were included. A random-effect meta-analysis of the 8 eligible studies was performed to summarize their results. The meta-analysis was based on the model of Dersimonian and Laird. The analysis found a reduction of risk of exacerbation (RR $0.76 \mathrm{CI} 0.63-092, \mathrm{I}^{2} 39 \%$ ), between the exacerbation rates observed in the FeNO-SC arm and SC arm (figure 2 ). The evidence on the usage of inhaled corticosteroids is based on a systematic review that found an approximately $20 \%$ average reduction in use of ICS when FeNO monitoring was applied in pediatrics patients (11-12)

\section{Transition Probabilities and utilities}

Utilities and transition probabilities of pediatric patients with mild-moderate asthma treated with inhaled corticosteroids as maintenance therapy of the model were extracted from a local previous study that evaluates the cost-utility of inhaled steroids in pediatric asthma within national guidelines for pediatric asthma (3, 13), Table 1 .

\section{Cost analysis:}

To estimate the cost of each health state defined in the model we extracted all costs of infants less than two years of age admitted on a tertiary center, in Medellin, Colombia due to asthma according to the national clinical guideline of asthma in children from January 2018 to December 2018. All costs and use of resources were collected directly from medical invoices and electronic medical records. The direct costs considered in the analysis include medical consultation at the emergency room, specialist referrals, chest physiotherapy, diagnosis support (laboratory, electrocardiogram, x-ray, etc), medication (oxygen, nebulization, antibiotics, corticosteroids, bronchodilators, etc.), medical devices, hotel services at intensive care unit, hotel services in a general medical ward, Table $\mathbf{2}$. Our country has been characterized by having a very small price variation in the last 10 years, especially in health services between the different clinics and over time (14). Moreover, the proportions of each of the costs remain relatively constant, which, with few variations in their composition in the last ten years (14). We use US dollars (Currency rate: US\$ $1.00=\operatorname{COP} \$ 3,000$ ) (15) to express all costs in the study. For the valuation of the indirect costs associated with the loss of productivity of parents, the human capital method was used, assuming everyone receives an income of at least a legal minimum wage for formal or informal work. The cost-opportunity of the productivity loss at the workplace and the caregiver was assessed based on the minimum wage without including the transportation assistance for the year 2019 (U\$229.81 per month). The legal minimum wage approved by the government 
was taken as a reference and not an average or median wage thereof, given that in Colombia more than $75 \%$ of the population has this value as income(16). Because all patients with asthma included were children, we assumed that at least one family, member accompanies the patient permanently during hospitalization, since pediatric hospitals in the country usually allow only one companion per patient in the hospital. The cost associated with transportation and food (does not include a stay), was assumed to correspond to $50 \%$ of the minimum wage per day.

\section{Sensitivity analyses}

To explore parameter uncertainty of the model inputs, a probabilistic sensitivity analysis was conducted by randomly sampling from each of the parameter distributions (beta distribution in the case of relative risk, and utilities, Dirichlet distribution for multinomial data in the case of transition probabilities, and gamma distribution in the case of costs). The expected costs and expected QALYs for each treatment strategy were calculated using that combination of parameter values in the model. This process was replicated one thousand times (i.e., second-order Monte Carlo simulation) for each treatment option resulting in the expected costutility. Decision uncertainty is represented in the cost-effectiveness acceptability frontiers, which plot the probability that the treatment strategy with the maximum expected net monetary benefit is the most cost-effective over a range of willingness-to-pay threshold values. Net monetary benefit was calculated by multiplying effect by societal willingness to pay and subtracting cost, with willingness to pay set at a ratio of US\$20,000 per QALY. We estimated the expected value of perfect information (EVPI). The EVPI is the maximum value that the health care system would be willing to pay for additional evidence to inform the reimbursement decision in the future. The population expected value of perfect information (PEVPI) was calculated to inform the expected cost of uncertainty (expected opportunity loss surrounding the decision) (17). Microsoft Exel @ was used in all analyzes.

\section{Results}

The model showed that FeNO-SC, was associated with lower total cost than SC (US $\$ 1333$ vs. US $\$ 1452$ average cost per patient), and higher QALYs (0.93 vs 0.92 average per patient); showing dominance. A position of dominance negates the need to calculate an incremental cost-effectiveness ratio (Table 2 ).

\section{Sensitivity analyses}

One-way showed that the probability of hospitalization has the highest impact on the outcome, but MS was the dominant strategy in all range of probabilities analyzed. The results of PSA are graphically represented in the cost-effectiveness plane, figure $3.53 .82 \%$ of simulations were graphed in quadrant 2 (lower cost, high QALYs), and $45.97 \%$ in quadrant 1 of this plane (high cost, high QALYs). The $95 \%$ CI for the cost per patient treated with FeNO-SC, and with SC were US\$ 1331 to 1335 and US\$ 1449 to 1454. The 95\% CI for QALYs per patient was 0.93 to 0.94 and 0.91 to 0.92 respectively. The net monetary benefits of FeNO-SC was higher than SC (US\$ 16591 vs US\$ 16885). The expected net benefit with perfect information US\$ 16885.9 and the expected value of perfect information were: US\$ 0.074. The Cost-effectiveness acceptability curve shows that the probability that FeNO-SC provides a more cost-effective use of resources compared with standard therapy exceeds $99 \%$ for all willingness to pay thresholds. The population expected value of perfect information (EVPIP) for the threshold of US\$20 was US\$ 1030015.

\section{Discussion}

Our study suggests that FeNO-SC achieving better outcomes at a lower cost over standard treatment without FeNO in children with mild to moderate allergic asthma. These better outcomes are due to reductions in the probabilities of asthma exacerbation and sub-optimal control of the disease, with the consequent increase of patients well controlled. The magnitude of annual cost savings for the health system (US\$ 118 per patient ) is no negligible if we consider that this disease affects between 10 to $13 \%$ of children and only $2.4 \%$ of them meet the criteria for total asthma control in Colombia. The findings constitute a new argument to include the FeNO-SC in clinical practice guidelines of pediatric asthma.

Our findings are in line with previous studies in the literature evaluating the economic value of FeNO of 
inserting FeNO monitoring into asthma management, some of them enrolling pediatric patients. Beerthuizen et al. assessed the cost-effectiveness of web-based monthly monitoring and of 4-monthly monitoring of FeNO as compared with standard care. The economic evaluation was performed alongside a multicentre RCT with a 1-year follow-up, and included 272 children aged between 4 and 18 years of age. The FeNO-based strategy had $83 \%$ chance of being most cost-effective at \euro40 000/QALY from a societal perspective (18). Berg et al. assessed the cost-effectiveness of FeNO measurement with NIOX MINO in the diagnosis of asthma and in optimizing asthma management using the expected reimbursement price of the device. In this study, the use of FeNO measurement in treatment decisions was less costly than asthma management based on standard guidelines (while in mild to severe patients, asthma management with FeNO measurement instead of standard guidelines resulted in cost-savings of $\backslash$ euro30 per patient and year, in more severe population, management with FeNO measurement would save costs of $\backslash$ euro160 per patient) and provided similar health benefits (7). Brooks et al. examined the impact of FeNO monitoring on the cost-effectiveness of asthma management compared with management without FeNO. FeNO in conjunction with current standard of care guidelines had decreased expected per-patient annual expenditure (US\$2,228) and increased expected perpatient annual QALYs (0.844) compared with current standard of care alone (US\$2,637 and 0.767)(9). Price et al. determined the cost-effectiveness of FeNO measurement using a hand-held monitor (NIOX MINO), at a reimbursement price of $£ 23$, for asthma diagnosis and management in the UK. Asthma management using FeNO measurement instead of lung function testing resulted in annual cost-savings of $£ 341$ and 0.06 QALYs gained for patients with mild to severe asthma and cost-savings of $£ 554$ and 0.004 QALYs gained for those with moderate to severe asthma(19). Sabatelli et al. evaluated the cost-effectiveness and budget impact of FeNO monitoring for management of adult asthma in Spain over a 1-year period. Adding FeNO to standard asthma care saved \euro62.53 per patient-year and improved QALYs by 0.026 per patient-year. The budget impact analysis revealed a potential net yearly saving of \euro129 million if FeNO monitoring had been used in primary care settings in Spain.8 Similarly, Harnan et al. assessed the cost-effectiveness of the hand-held electrochemical devices NIOX MINOß (Aerocrine, Solna, Sweden), NIOX VEROß (Aerocrine) and NO breath@ (Bedfont Scientific, Maidstone, UK) for the diagnosis and management of asthma. The de novo management model indicated that the ICER of guidelines plus FeNO monitoring using NO breath compared with guidelines alone in children is expected to be approximately $£ 45,200$ per QALY gained, concluding that FeNO-guided management has the potential to be cost-effective, although this is largely dependent on the duration of effect(10)

The last version of the Global Initiative for Asthma refers to children "FeNO-guided treatment significantly reduces exacerbation rates compared with guidelines-based treatment (Evidence A). However, further studies are needed to identify the populations most likely to benefit from FeNO-guided treatment, and the optimal frequency of FeNO monitoring"(2). However, in the references that support this statement included only RCTs and not economic evaluations were revised. The dynamics between clinical research in the effectiveness and research of efficiency of the allocation of health resources must be coordinated, synchronous of the moment to make a recommendation from individual to public health level. It is clear the complexity of the transferability of economics evaluations, but this situation highlights the need to assess the health technologies in the clinical guidelines no only evaluating effectiveness or safety but also review economical topics to increase the level of recommendation in clinical guidelines.

A very important aspect of our model is that it was robust to changing the values of the model's utilities, probabilities, and costs using oneway and probabilistic sensibility analysis of a Markov model. FeNO-SC was always the cost-effectiveness strategy in all ranges of values of utilities, probabilities, and costs. Even more, FeNO-SC was always the cost-effectiveness strategy in all ranges of thresholds evaluated with a low population EVPI. These findings in the sensibility analysis have cardinal importance in our study because many of the inputs were extracted from literature, which all were hospital-based and undertaken in affluent countries; also allow decision making with an estimated degree of uncertainty in each cost parameter or QALYs per strategy.

Our study has some limitations. The cost data were collected retrospectively. Asthma treatment and the costs in question, including hospital prices, did not markedly change to our days. Furthermore, our country 
has been characterized by having a very small price variation in the last 10 years, especially in health services (14). Additionally, we use utilities extracted from the literature and not estimated directly from our population. As was mentioned previously, the reliability and robustness of the results were evaluated by sensitivity analyses.

In conclusion, the FeNO-SC was cost-effective for infant with asthma moderate or severe. Our study provides evidence that should be used by decision-makers to improve clinical practice guidelines and should be replicated to validate their results in other middle-income countries.

\section{References}

1. Neffen H, Fritscher C, Schacht FC, Levy G, Chiarella P, Soriano JB, et al. Asthma control in Latin America: the Asthma Insights and Reality in Latin America (AIRLA) survey. Rev Panam Salud Publica. 2005;17(3):191-7.

2. Global Iniciative for Asthma Management and Prevention 2020 [Available from: www.ginaasthma.org .

3. Colciencias MdSyPS-. Guia de Practica Clinica Para el diagnostico, atencion integral y seguimiento de ninos y ninas con diagnostico de Asma. Guia No. 012014 [Available from: ,http://gpc.minsalud.gov.co/gpc-sites/Repositorio/Conv_500/GPC_asma/GPC_Comp_Asma.pdf .

4. Silvestri M, Sabatini F, Spallarossa D, Fregonese L, Battistini E, Biraghi MG, et al. Exhaled nitric oxide levels in non-allergic and allergic mono- or polysensitised children with asthma. Thorax. 2001;56(11):857-62.

5. Strunk RC, Szefler SJ, Phillips BR, Zeiger RS, Chinchilli VM, Larsen G, et al. Relationship of exhaled nitric oxide to clinical and inflammatory markers of persistent asthma in children. J Allergy Clin Immunol. 2003;112(5):883-92.

6. Petsky HL, Kew KM, Chang AB. Exhaled nitric oxide levels to guide treatment for children with asthma. Cochrane Database Syst Rev. 2016;11:CD011439.

7. Berg J, Lindgren P. Economic evaluation of FE(NO) measurement in diagnosis and 1-year management of asthma in Germany. Respir Med. 2008;102(2):219-31.

8. Sabatelli L, Seppala U, Sastre J, Crater G. Cost-effectiveness and Budget Impact of Routine Use of Fractional Exhaled Nitric Oxide Monitoring for the Management of Adult Asthma Patients in Spain. J Investig Allergol Clin Immunol. 2017;27(2):89-97.

9. Brooks EA, Massanari M. Cost-Effectiveness Analysis of Monitoring Fractional Exhaled Nitric Oxide (FeNO) in the Management of Asthma. Manag Care. 2018;27(7):42-8.

10. Harnan SE, Tappenden P, Essat M, Gomersall T, Minton J, Wong R, et al. Measurement of exhaled nitric oxide concentration in asthma: a systematic review and economic evaluation of NIOX MINO, NIOX VERO and NObreath. Health Technol Assess. 2015;19(82):1-330.

11. Ralston SL, Lieberthal AS, Meissner HC, Alverson BK, Baley JE, Gadomski AM, et al. Clinical practice guideline: the diagnosis, management, and prevention of bronchiolitis. Pediatrics. 2014;134(5):e1474-502.

12. Morphew T, Shin HW, Marchese S, Pires-Barracosa N, Galant SP. Phenotypes favoring fractional exhaled nitric oxide discordance vs guideline-based uncontrolled asthma. Ann Allergy Asthma Immunol. 2019;123(2):193-200.

13. Rodriguez-Martinez CE, Sossa-Briceno MP, Castro-Rodriguez JA. Cost-utility analysis of the inhaled steroids available in a developing country for the management of pediatric patients with persistent asthma. J Asthma. 2013;50(4):410-8.

14. Estadisticas DAN. Indice de Precios al Consumidor - IPC 2020 [Available from:https://www.dane.gov.co/index.php/estadisticas-por-tema/precios-y-costos/indice-de-precios-alconsumidor-ipc 
15. la Bd, Republica. Tasa Representativa del Mercado (TRM - Peso por dolar) 2019 [cited 2020. Available from:https://www.banrep.gov.co/es/estadisticas/trm.

16. Departamento, Nacional, (DANE) DE. Archivo Nacional de Datos 2019 [Available from:https://sitios.dane.gov.co/anda-index/.

17. Felli JC, Hazen GB. Sensitivity analysis and the expected value of perfect information. Med Decis Making. 1998;18(1):95-109.

18. Beerthuizen T, Voorend-van Bergen S, van den Hout WB, Vaessen-Verberne AA, Brackel HJ, Landstra AM, et al. Cost-effectiveness of FENO-based and web-based monitoring in paediatric asthma management: a randomised controlled trial. Thorax. 2016;71(7):607-13.

19. Price D, Berg J, Lindgren P. An economic evaluation of NIOX MINO airway inflammation monitor in the United Kingdom. Allergy. 2009;64(3):431-8.

20. Buendia JA. [Attitudes, knowledge and beliefs of patient about anti-hypertensive drugs]. Biomedica. 2012;32(4):578-84.

\section{Abbreviations}

Fractional exhaled nitric oxide (FeNO)

$\mathrm{SC}$ with FeNO monitoring to guide asthma management in Children (FeNO-SC)

quality-adjusted life years (QALYs)

randomized clinical trials $(\mathrm{RCT})$

Acknowledgements : none

Funding: Own fundings

Conflict of interest statement for all authors : All authors declare that they do not have any conflict of interest in this publication.

\section{Hosted file}

Table 1.docx available at https://authorea.com/users/316889/articles/456400-cost-utility-offractional-exhaled-nitric-oxide-monitoring-for-the-management-of-children-asthma

\section{Hosted file}

Table 2.docx available at https://authorea.com/users/316889/articles/456400-cost-utility-offractional-exhaled-nitric-oxide-monitoring-for-the-management-of-children-asthma

\section{Hosted file}

Table 3.docx available at https://authorea.com/users/316889/articles/456400-cost-utility-offractional-exhaled-nitric-oxide-monitoring-for-the-management-of-children-asthma 

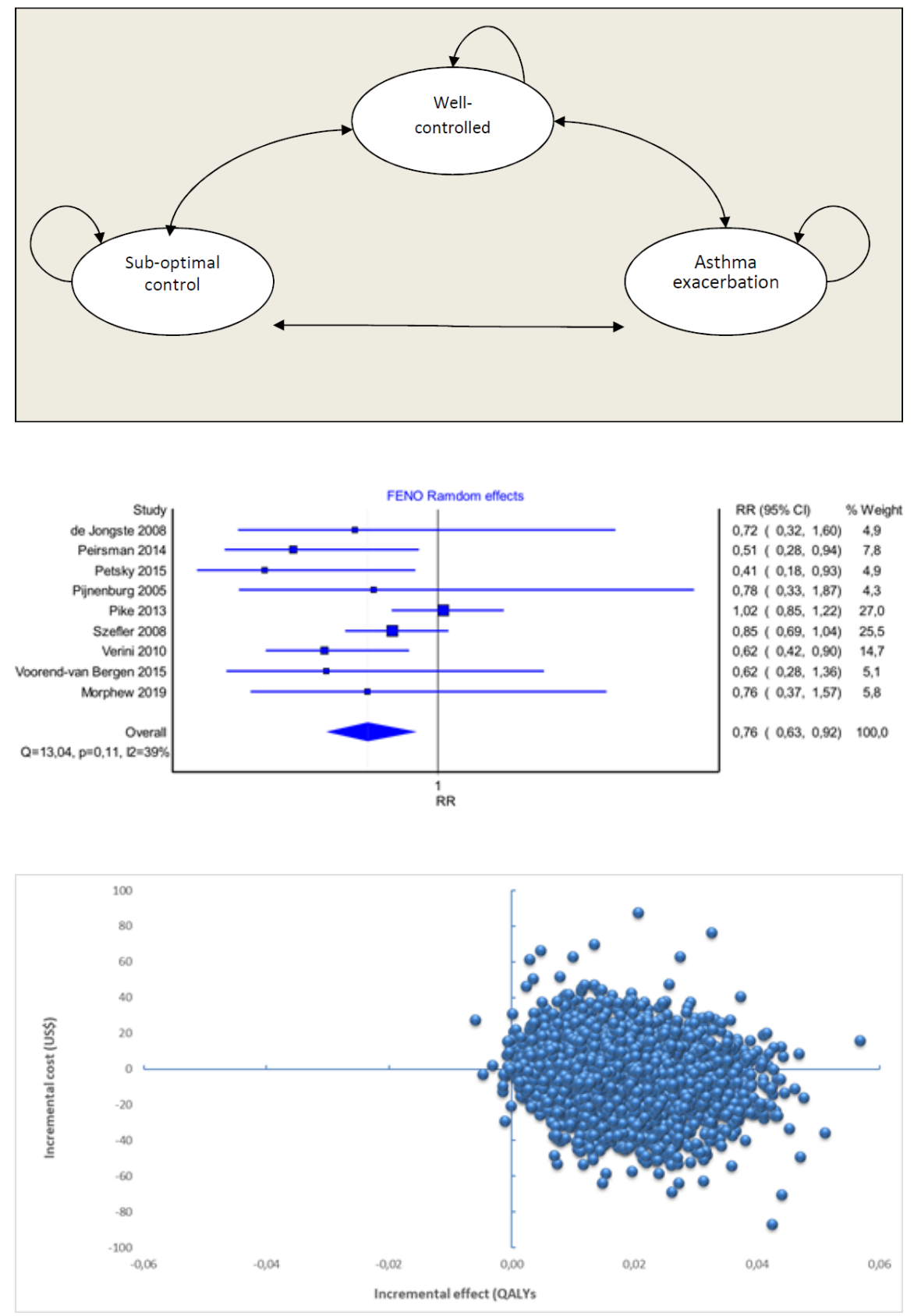